\title{
Damped oscillations of the probability of random events followed by absolute refractory period
}

\author{
A.V. Paraskevov ${ }^{1,2}$, A.S. Minkin ${ }^{1}$ \\ ${ }^{1}$ National Research Centre "Kurchatov Institute", 123182 Moscow, Russia \\ ${ }^{2}$ Moscow Institute of Physics and Technology, 141701 Dolgoprudny, Russia
}

\begin{abstract}
Many events are followed by an absolute refractory state, when for some time after the event a repetition of a similar event is impossible. If uniform events, each of which is followed by the same period of absolute refractoriness, occur randomly, as in the Bernoulli scheme, then the event probability as a function of time can exhibit damped transient oscillations caused by a specific initial condition. Here we give an exact analytical description of the oscillations, with a focus on application within neuroscience. The resulting formulas stand out for their relative simplicity, enabling analytical calculation of the damping coefficients for the second and third peaks of the event probability.
\end{abstract}

Keywords: renewal point process, absolute refractory period, damped oscillations, neuron, stochastic spiking

\section{Introduction}

Many natural and technical events are followed by a refractory period, when for some time after the event a repetition of a similar event is unlikely (relative refractory period) or even impossible (absolute refractory period). A characteristic natural example is the neuron and the refractory nature of its ability to generate electrical impulses - spikes. In turn, a typical example from the technique is the existence of so-called dead time for some types of detectors (so-called the Type 1 counters [1]), especially for photodetectors. The dead time is a fixed period of time after the detector triggering during which it becomes inoperative. Both the neuronal refractoriness and the detector dead time influence essentially counting distributions. If instant events of the same type, each of which is followed by the same absolute refractory period $\tau_{r e f}$, occur randomly, the average interval $T$ between the events can be represented as the sum $T=\tau_{\text {ref }}+T_{0}$, where $T_{0}$ is the value of the average interval if refractoriness is absent. A more nontrivial consequence of the refractory period is damped oscillations of the event occurrence probability as a function of time [2-4], which are strongly pronounced at $T_{0} \lesssim \tau_{\text {ref }}$. Such transient oscillations arise due to (i) a specific initial condition and (ii) the fact that random instant events ordered in time in the presence 
of refractoriness become dependent on each other. In particular, the probability of a subsequent event depends on the time elapsed from the preceding event. Random point processes of this kind are called renewal processes and are the subject of study of the renewal theory [1]. This theory has a method of getting an explicit analytical description for the damped oscillations of the event probability by finding of so-called renewal density $[1,2]$. For instance, this has been repeatedly done for the classic example of the Poisson process modulated by the dead time or absolute refractory period [5-16]. However, the resulting analytical formula for the time-dependent probability of the event is quite cumbersome, and that complicates further analysis.

In this paper, for the Bernoulli process modulated by absolute refractoriness we give a compact analytical description of the damped oscillations without invoking the renewal theory. The description is presented in four equivalent forms (three kinds of a recurrence formula and one explicit formula) and is quantitatively consistent with both the results of numerical simulations and those of the renewal theory. One kind of the recurrence formula is especially simple, enabling accurate analytical calculation of the damping coefficients. Surprisingly, these are quite robust against changing the values of the model parameters.

Finally, ready-to-use MATLAB/Octave codes for performing simulations and plotting the graphs of the obtained formulas are included as supplementary material.

\section{Formulation of the model problem}

For certainty, consider a model neuron that stochastically emits spikes, each of which is followed by an absolute refractory period. In particular, the neuron can spontaneously emit a spike with probability $p_{s}$ per unit time (i.e., in a given elementary time interval $\Delta t$ ) so that after the spike emission the neuron becomes temporarily inactive, i.e. it cannot emit spikes during the refractory period $\tau_{r e f}=n_{r e f} \triangle t$, where $n_{r e f}$ is a positive integer. Then the mean rate of occurrence of events in the absence of refractoriness $\nu_{0}=1 / T_{0}=p_{s} / \triangle t$, and at $\tau_{\text {ref }} \neq 0$ from the equality $T=\tau_{r e f}+T_{0}$ for the mean rate $\nu=1 / T$ one gets $\nu=\nu_{0} /\left(1+\tau_{\text {ref }} \nu_{0}\right)$. In fact, this mean rate is equal to the ratio of the total number of spikes to observation time $T_{o b s}$, given that $T_{o b s}>>\tau_{r e f}, T_{0}$. It is also useful to introduce the asymptotic value of the average probability of spike generation at each step, $\bar{p}_{s}=\nu \Delta t=p_{s} /\left(1+n_{r e f} p_{s}\right)$, such that, by the analogy with the formula for $\nu_{0}$,

$$
\nu=\bar{p}_{s} / \triangle t=p_{s} /\left(\triangle t+p_{s} \tau_{r e f}\right)
$$

An algorithm for simulating the neuron's dynamics is extremely simple and as follows. Dividing the observation interval $T_{o b s}$ by $N$ equal steps $\triangle t, T_{o b s}=N \triangle t$, these time steps are numbered 
by a sequence of natural numbers starting with 1 . At each step, a random number $\xi$, uniformly distributed from zero to one, is generated and compared with the given probability $p_{s}$ of generating a spike. If $\xi \leq p_{s}$, it is assumed that spike has been generated at this time step. After the spike generation, the neuron cannot emit a next spike during refractory period $\tau_{r e f}=n_{r e f} \triangle t$. The event of spike generation at an arbitrary $k$-th step is further denoted by $A_{k}$. For definiteness, the initial state of the neuron is chosen as a moment when the neuron has just left the refractory state after emitting a spike.

Performing either a large number of repeated passes of the observation interval $T_{o b s}$ for a single neuron or a single pass for the large ensemble of independent neurons, one gets a statistical distribution of the occurrence of events in the entire sequence of $N$ time intervals. Normalizing this distribution by the number of either the passes or the neurons in the ensemble, one obtains the probability distribution $P_{k}$ of spike generation at $k$-th elementary time step of the observation interval. Due to the refractory period, the event probability, as a function of time, exhibits damped oscillations with the average period equal to $\tau_{\text {ref }}$ (Fig. 1).

In turn, the analytical problem consists in finding the probability $P_{k} \equiv P\left(A_{k}\right)$ of spike generation at each $k$-th elementary time step so that in the asymptotic limit $k \rightarrow \infty$ one would obtain $P_{k} \rightarrow \bar{p}_{s}$.

\section{Derivation of the exact analytical formula for $P_{k}$}

The probability of spike generation at $k$-th time step $(k=\overline{1, N}), P_{k}=P\left(A_{k}\right)$, is equal to the product of $p_{s}$ and the probability that in the interval from $k-n_{\text {ref }}$ to $k-1$ inclusively no spike was emitted,

$$
P_{k}=p_{s}\left[1-P\left(A_{k-n_{r e f}}+A_{k-n_{r e f}+1}+\ldots+A_{k-1}\right)\right]
$$

Spike generation events in $n_{\text {ref }}$ consecutive time intervals are pairwise incompatible events. Therefore, according to the summation theorem for the probabilities of pairwise incompatible events, the probability of the sum in Eq. (2) equals the sum of probabilities

$$
P\left(A_{k-n_{r e f}}+A_{k-n_{r e f}+1}+\ldots+A_{k-1}\right)=\sum_{j=k-n_{r e f}}^{k-1} P\left(A_{j}\right),
$$

and the sought-for probability at the $k$-th step is determined in a recurrent manner, with the recursion period equal to the refractory period,

$$
P_{k}=p_{s}\left(1-\sum_{j=k-n_{r e f}}^{k-1} P_{j}\right)=p_{s}\left[1-\left(S_{k-1}-S_{k-n_{r e f}-1}\right)\right],
$$



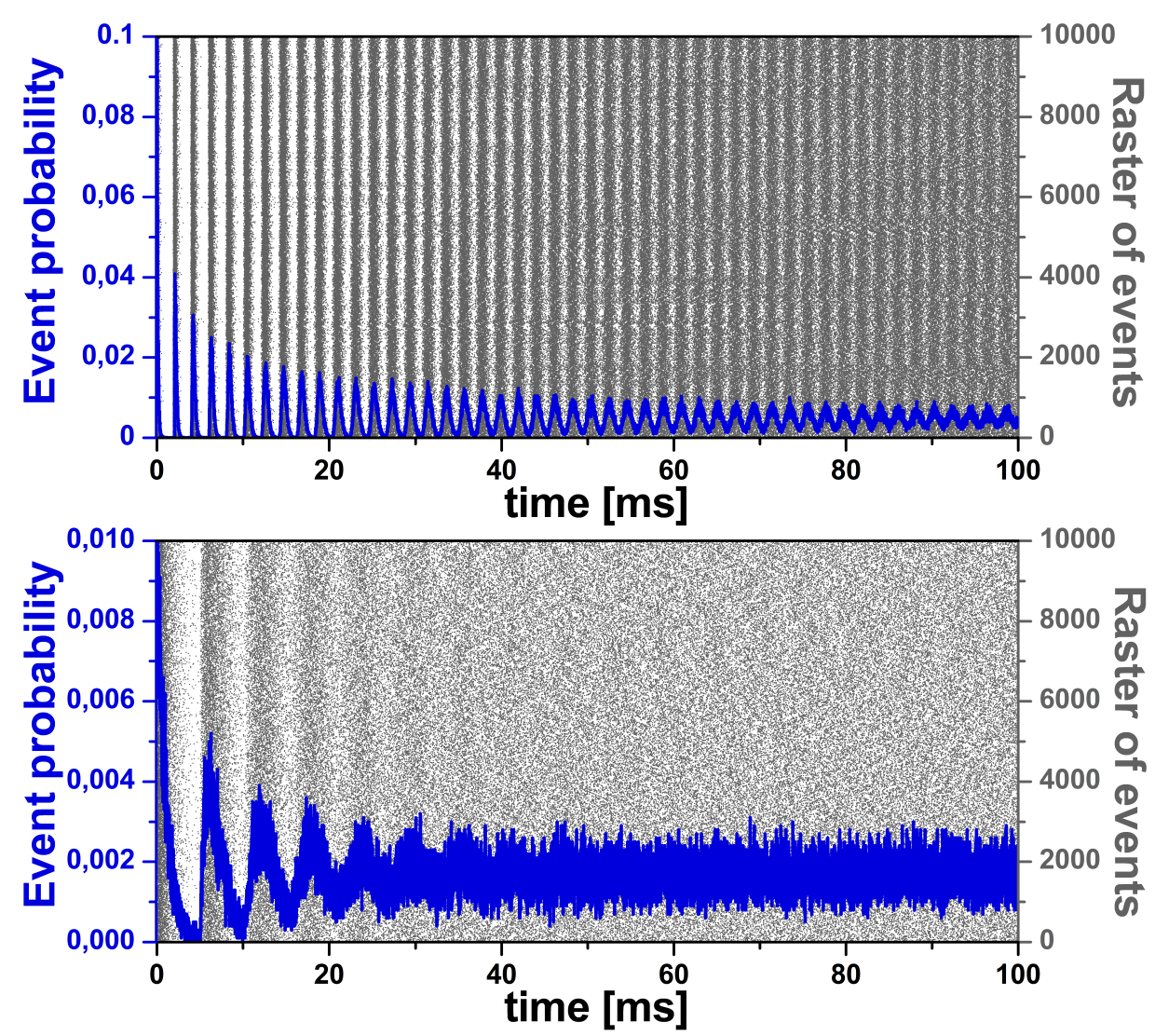

Figure 1: Numerical simulation of time dependence of the event probability, where an event is spike generation, for $10^{4}$ disconnected stochastically-spiking neurons at time step $\triangle t=0.01 \mathrm{~ms}$. Top: Raster of events (gray dots, scale on the right) and the corresponding time dependence of the statistical probability of an event (blue line, scale on the left) at $p_{s}=0.1$ and $\tau_{r e f}=2 \mathrm{~ms}$. It is seen that the period of damped oscillations of probability is $\tau_{r e f}$. The asymptotic probability value to which the damped oscillations converge corresponds to the calculated value $\bar{p}_{s}=4.76 \cdot 10^{-3}$ (or the average event frequency $\nu=476 \mathrm{~Hz}$, see (1)). Bottom: Similar graphs for $p_{s}=0.01$ and $\tau_{r e f}=5 \mathrm{~ms}$. For this case, $\bar{p}_{s}=1.67 \cdot 10^{-3}$ and $\nu=167 \mathrm{~Hz}$. Note that the parameters of the neuron model (in particular, nonphysiologically large value of $p_{s}$ ) are chosen solely for the illustrative purpose.

where, by definition,

$$
S_{m}=\left\{\begin{array}{l}
0, m \leq 0, \\
\sum_{j=1}^{m} P_{j}, m>0 .
\end{array}\right.
$$

Such a definition of $S_{m}$ allows us to directly generalize the formula for $P_{k}$ to the range $1 \leq k \leq n_{\text {ref }}$. The closed formula for $S_{k}$ has the form of a linear recurrent sequence

$$
S_{k}=P_{k}+S_{k-1}=p_{s}\left(1+S_{k-n_{r e f}-1}\right)+\left(1-p_{s}\right) S_{k-1}
$$



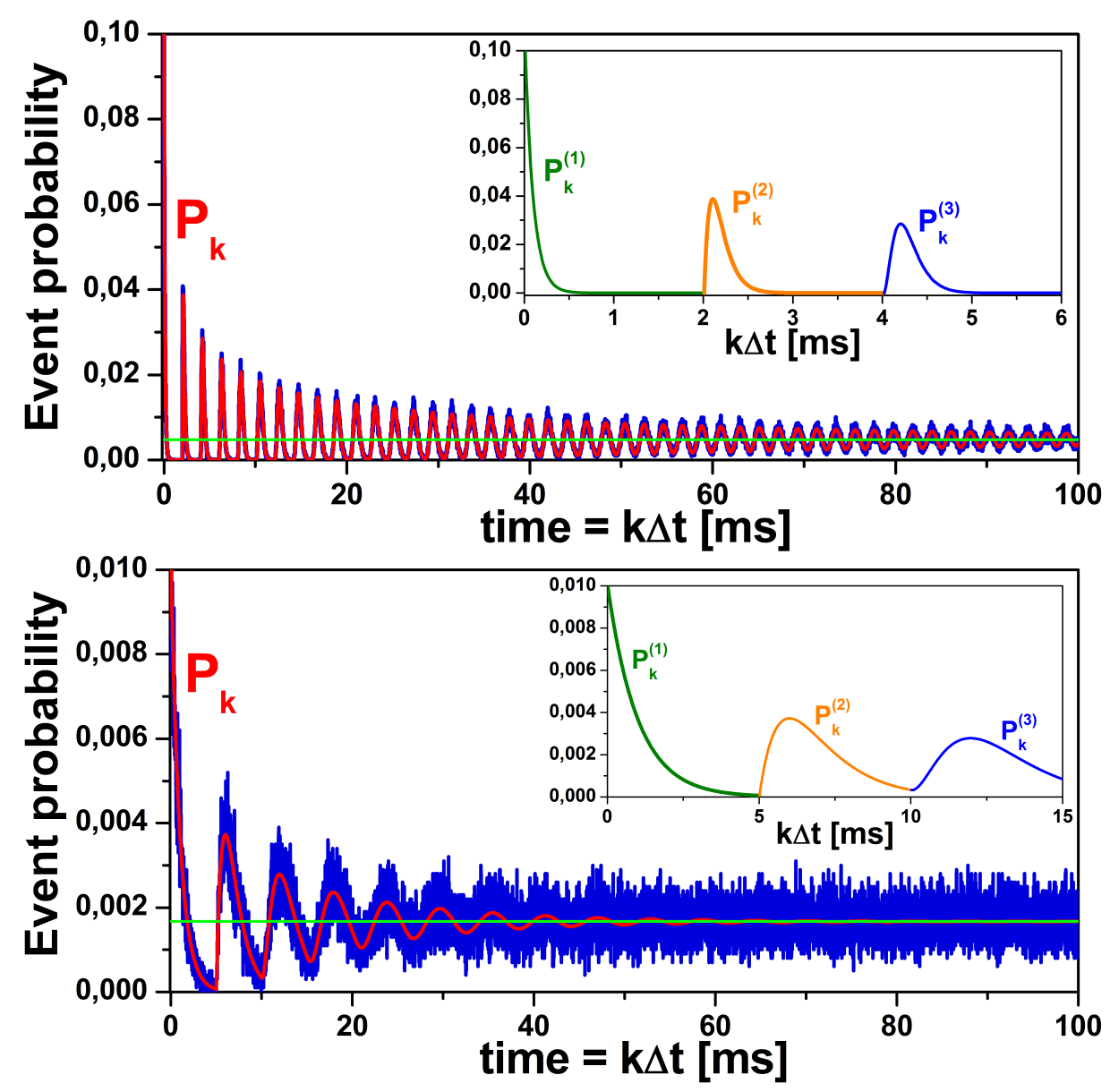

Figure 2: Comparison of the results of numerical simulations (see Fig. 1) with the calculation by mutually equivalent analytical formulas (4), (9), (15), (20) and (21). Top: Time dependence of the statistical probability of an event (blue line, data taken from the top panel of Fig. 1) and the corresponding analytic curve $P_{k}$ (red line) at $p_{s}=0.1$ and $\tau_{r e f}=2 \mathrm{~ms}$. The asymptotic value $P_{\infty}=\bar{p}_{s}=4.76 \cdot 10^{-3}$ is shown by the green horizontal line. Inset: Partial analytical curves for the first three refractory intervals calculated by formulas (7), (10) and (12). Bottom: Similar graphs (main graph and inset) for the parameter values $p_{s}=$ 0.01 and $\tau_{\text {ref }}=5 \mathrm{~ms}$, giving the asymptotic probability $P_{\infty}=1.67 \cdot 10^{-3}$.

The resulting formula (4) accurately describes the numerical statistics (Fig. 2).

One should note three important consequences.

First, for $1 \leq k \leq n_{\text {ref }}+1$, where

$$
S_{k}=p_{s}+\left(1-p_{s}\right) S_{k-1}
$$

the sum $S_{k}$ can be easily found explicitly, as it is an arithmetic-geometric progression of the form

$$
S_{k}=r S_{k-1}+d,
$$


where $S_{1}=p_{s}, r=1-p_{s}$ и $d=p_{s}$. According to the formula for the explicit form of the $k$-th term of this progression,

$$
S_{k}=r^{k-1}\left[S_{1}+\frac{d}{r-1}\right]-\frac{d}{r-1}=1-\left(1-p_{s}\right)^{k}
$$

Hence, the probability of spike generation in the interval $1 \leq k \leq n_{\text {ref }}+1$ is equal to

$$
P_{k} \equiv P_{k}^{(1)}=p_{s}\left[1-S_{k-1}\right]=p_{s}\left(1-p_{s}\right)^{k-1}
$$

Here and below, the upper index in parentheses indicates the number of the refractoriness interval, counted from the initial moment $t=0$. Given the initial condition, this probability naturally coincides with the probability of the first spike generation at an arbitrary $k$-th step, such that $\sum_{k=1}^{\infty} P_{k}^{(1)}=1$, and refers to the geometric distribution.

Second, the formula (4) makes it easy to obtain the asymptotic probability value $P_{k}$ at $k \rightarrow \infty$. The difference $S_{k-1}-S_{k-n_{r e f-1}}$ contains $n_{r e f}$ terms. At $k \rightarrow \infty$ the probability at the $k$-th step remains practically unchanged. Denoting it as $P_{\infty}$, from the general formula (4) one gets

$$
P_{\infty}=p_{s}\left[1-n_{\text {ref }} P_{\infty}\right]
$$

whence

$$
P_{\infty}=p_{s} /\left(1+n_{r e f} p_{s}\right)
$$

Third, calculating the adjacent terms $P_{k+1}$ or $P_{k-1}$ similarly to the formula (6), one can exclude sums (5) from the formula (4) and obtain a linear recurrent sequence for $P_{k}$ :

$$
P_{k}=\left\{\begin{array}{l}
p_{s}\left(1-p_{s}\right)^{k-1}, k \leq n_{\text {ref }}+1, \\
p_{s} P_{k-n_{r e f}-1}+\left(1-p_{s}\right) P_{k-1}, k>n_{\text {ref }}+1 .
\end{array}\right.
$$

This formula is completely equivalent with Eq. (4) and using it one can easily find the expression for $P_{k}$ in an explicit form within the intervals of $k$ multiples of $n_{r e f}+1$.

For example, at $n_{\text {ref }}+1 \leq k \leq 2\left(n_{\text {ref }}+1\right)$ one gets

$$
P_{k}^{(2)}=\left(k-n_{\text {ref }}-1\right) p_{s}^{2}\left(1-p_{s}\right)^{k-n_{r e f}-2}+P_{k}^{(1)}=P_{k}^{(1)}\left[1+\left(k-n_{r e f}-1\right) q\right],
$$

where $q=p_{s}\left(1-p_{s}\right)^{-\left(n_{\text {ref }}+1\right)}$.

Next, at $2\left(n_{\text {ref }}+1\right) \leq k \leq 3\left(n_{\text {ref }}+1\right)$ one gets

$$
\begin{aligned}
P_{k}^{(3)} & =\frac{1}{2}\left(k-2 n_{\text {ref }}-2\right)\left(k-2 n_{\text {ref }}-1\right) p_{s}^{3}\left(1-p_{s}\right)^{k-2 n_{r e f}-3}+P_{k}^{(2)}= \\
& =P_{k}^{(1)}\left[1+\left(k-n_{\text {ref }}-1\right) q+\frac{1}{2}\left(k-2 n_{\text {ref }}-2\right)\left(k-2 n_{\text {ref }}-1\right) q^{2}\right] .
\end{aligned}
$$


It is worth noting that the numerical coefficient in the highest-order term with respect to $q$ is the so-called triangular number $j(j+1) / 2$ at $j=k-2 n_{\text {ref }}-2$. The inset in Fig. 2 shows the plots for $P_{k}^{(1)}, P_{k}^{(2)}$ and $P_{k}^{(3)}$.

Using the induction method, one can obtain a formula for $P_{k}^{(m+1)}$, valid within the range $m\left(n_{\text {ref }}+1\right) \leq k \leq(m+1)\left(n_{\text {ref }}+1\right)$ :

$$
P_{k}^{(m+1)}=\left[\frac{1}{m !} \prod_{j=1}^{m}\left(k-m \cdot n_{r e f}-j\right)\right] q^{m} P_{k}^{(1)}+P_{k}^{(m)} .
$$

The general explicit expression for $P_{k}$ in the polynomial form is as follows:

$$
\begin{aligned}
P_{k} & =P_{k}^{(1)}\left[1+a_{k}^{(1)} p_{s}\left(1-p_{s}\right)^{-\left(n_{r e f}+1\right)}+a_{k}^{(2)} p_{s}^{2}\left(1-p_{s}\right)^{-2\left(n_{r e f}+1\right)}+\ldots\right]= \\
& =P_{k}^{(1)}\left[1+\sum_{i=1}^{m} a_{k}^{(i)} q^{i}\right],
\end{aligned}
$$

where $m$ is the integer part of the rational number $k / n_{\text {ref }}$ rounded off to a smaller value, $q=$ $p_{s}\left(1-p_{s}\right)^{-\left(n_{r e f}+1\right)}$, and the coefficients

$$
a_{k}^{(m)} \equiv \frac{\theta\left(k-m\left(n_{r e f}+1\right)\right)}{m !} \prod_{j=1}^{m}\left(k-m \cdot n_{r e f}-j\right),
$$

where the unit step function $\theta(x)=1$ at $x \geq 0$ and $\theta(x)=0$ otherwise.

Taking into account the equality

$$
\frac{1}{m !} \prod_{j=1}^{m}(n-j)=\frac{(m+1)}{n} C_{n}^{m+1}=C_{n-1}^{m}, n>m \geq 1,
$$

where $C_{n}^{k}$ is the standard binomial coefficient,

$$
C_{n}^{k}=\frac{n !}{k !(n-k) !}=\left(\begin{array}{l}
n \\
k
\end{array}\right)
$$

the coefficients $a_{k}^{(m)}$ are directly expressed through the binomial coefficients:

$$
a_{k}^{(m)}=C_{k-m \cdot n_{r e f}-1}^{m} \theta\left(k-m\left(n_{r e f}+1\right)\right)
$$

Another equivalent formula for $P_{k}$ can be found in a different way, as follows. Denote $P\left(A_{k} \mid A_{j}\right)$ the conditional probability of spike generation at the $k$-th step, provided that the previous spike was generated at the $j$-th step. At times greater than the refractory period, i.e. at $k>n_{r e f}$, the probability of generating a subsequent spike depends only on the moment of a previous spike. Therefore, taking into account the initial condition, $P\left(A_{k} \mid A_{j}\right)=P_{k-j-n_{r e f}}^{(1)}$, if $k-j>n_{r e f}$, and $P\left(A_{k} \mid A_{j}\right)=0$, if $k-j \leq n_{r e f}$. 
At $k>n_{\text {ref }}+1$, the probability $P_{k}$ can be written as the sum of two terms: the probability $P_{k}^{(1)}$ that a spike will be emitted for the first time at the $k$-th step and the probability that at least one spike has been emitted previously. The latter has the form of a convolution and follows from the total probability formula.

$$
P_{k}=P_{k}^{(1)}+\sum_{j=1}^{k-1} P_{j} \cdot P\left(A_{k} \mid A_{j}\right)=P_{k}^{(1)}+\sum_{j=1}^{k-n_{r e f}-1} P_{j} \cdot P_{k-j-n_{r e f}}^{(1)} .
$$

Notably, using substitution $i=k-j-n_{\text {ref }}$, one can virtually swap the indices of the multipliers under the sign of the sum in (20), while the formula does not change its numerical value:

$$
P_{k}=P_{k}^{(1)}+\sum_{i=1}^{k-n_{r e f}-1} P_{k-i-n_{r e f}} \cdot P_{i}^{(1)} .
$$

Despite the different appearance in relation to the formulas (4) and (9), the formulas (20), (21) lead to the same numerical results and can be derived from the recurrent formula (9). In particular, the formula (21) can be straightforwardly obtained from (9) by successively substituting in the latter the values $P_{k-1}, P_{k-2}, \ldots, P_{k-n_{r e f}-1}$ and taking into account the definition (7) for $P_{k}^{(1)}$.

\section{Damping of the oscillations}

The formulas (10) and (12) allow one to analytically calculate the relative damping of the second and third peaks of $P_{k}$. Finding the location of these peaks corresponds to solving a linear and quadratic algebraic equation, respectively. In particular, for $\left(n_{r e f}+1\right) \leq k \leq 2\left(n_{\text {ref }}+1\right)$, calculating $d P_{k}^{(2)} / d k=0$ by the explicit formula (10) we get the location of the second peak,

$$
k_{\max 2}=n_{r e f}+1+R
$$

where

$$
\begin{aligned}
R & =1 / u-1 / q, \\
q & =p_{s}\left(1-p_{s}\right)^{-\left(n_{r e f}+1\right)}, \\
u & =\ln \frac{1}{\left(1-p_{s}\right)} .
\end{aligned}
$$

Substituting $k_{\max 2}$ into Eq. (10), we get the amplitude of the second peak

$$
P_{\max }^{(2)} \equiv P_{k_{\max 2}}^{(2)}=P_{k_{\max 2}}^{(1)} \frac{q}{u}=\frac{p_{s}^{2}}{u}\left(1-p_{s}\right)^{R-1} .
$$

The damping can be traced simply by the ratio of amplitudes for the adjacent peaks,

$$
D_{i+1}=P_{\max }^{(i+1)} / P_{\max }^{(i)} .
$$


Given that $k_{\max 1}=1$ and $P_{k_{\max 1}}^{(1)}=p_{s}$, for the second peak we get

$$
D_{2}=\frac{p_{s}}{u}\left(1-p_{s}\right)^{R-1}
$$

For the third refractory interval, $2\left(n_{\text {ref }}+1\right) \leq k \leq 3\left(n_{\text {ref }}+1\right)$, calculation of $d P_{k}^{(3)} / d k=0$ by the formula (12) results in a quadratic equation,

$$
x k^{2}-y k-z=0,
$$

where

$$
\begin{aligned}
& x=\frac{1}{2} q^{2} u \\
& y=q^{2}+\left(2 n_{r e f}+\frac{3}{2}\right) q^{2} u-q u \\
& z=-\left(2 n_{r e f}+\frac{3}{2}\right) q^{2}-\left(n_{r e f}+1\right)\left(2 n_{r e f}+1\right) q^{2} u+q+\left(n_{r e f}+1\right) q u-u .
\end{aligned}
$$

A suitable solution of this equation is the root

$$
k_{\max 3}=\frac{y+\sqrt{y^{2}+4 x z}}{2 x}=2\left(n_{r e f}+1\right)+R+X,
$$

where

$$
X=-\frac{1}{2}+\sqrt{\frac{1}{4}+\frac{1}{u^{2}}-\frac{\left(2 n_{r e f}+1\right)}{q}-\frac{1}{q^{2}}} .
$$

Substituting $k_{\max 3}$ into the formula $(12)$ for $P_{k}^{(3)}$ yields

$$
P_{k_{\max 3}}^{(3)}=P_{k_{\max 3}}^{(1)}\left[1+\left(n_{r e f}+1+R+X\right) q+\frac{1}{2}(R+X)(1+R+X) q^{2}\right] .
$$

After elementary but cumbersome calculations one can get a compact analytical expression for the damping coefficient of the third peak,

$$
D_{3}=P_{k_{\max 3}}^{(3)} / P_{k_{\max 2}}^{(2)}=p_{s}\left(1-p_{s}\right)^{X} \cdot\left(\frac{1}{u}+X+\frac{1}{2}\right)
$$

Numerical calculations have confirmed the validity of the obtained formulas. Below we have also listed the numerical values of the relevant quantities, calculated by the above formulas, for the two examples shown in Figs. 1 and 2.

For the first example, at $\tau_{\text {ref }}=2 \mathrm{~ms}\left(n_{\text {ref }}=\tau_{\text {ref }} / \triangle t=200\right.$ at $\left.\triangle t=0.01 \mathrm{~ms}\right)$ and $p_{s}=0.1$, we get $q \approx 1.6 \times 10^{8}, u \approx 0.1, R \approx 9.5, k_{\max 2}=210, P_{k_{\max 2}}^{(2)} \approx 0.04, k_{\max 3}=420, P_{k_{\max 3}}^{(3)} \approx 0.03$, $D_{2} \approx 0.39$, and $D_{3} \approx 0.74$. 
For the second example, at $\tau_{\text {ref }}=5 \mathrm{~ms}\left(n_{\text {ref }}=500\right)$ and $p_{s}=0.01$, we get $q \approx 1.5, u \approx 0.01$, $R \approx 99, k_{\max 2}=599, P_{k_{\max 2}}^{(2)} \approx 0.004, k_{\max 3}=1196, P_{k_{\max 3}}^{(3)} \approx 0.003, D_{2} \approx 0.37$, and $D_{3} \approx 0.75$.

It is seen that the numerical values $D_{2} \approx 0.4$ and $D_{3} \approx 0.75$ are fairly robust against changing the parameters. Thus, the magnitude of the second peak is approximately equal to $40 \%$ of the magnitude of the first. In turn, the value of the third peak is approximately $75 \%$ of the value of the second or $30 \%$ of the value of the first peak.

\section{Comparison with the renewal theory}

In the framework of the renewal theory $[1,2]$, when the intervals between events are independent random variables, knowing the distribution density of such intervals, one can find the time dependence of the event probability, provided that an event occurred at the initial moment in time. In particular, the sought-for probability is expressed through the so-called renewal density $h(t)$,

$$
P_{k}^{(r d)}=h(k \triangle t) \triangle t
$$

In turn, the renewal density $h(t)$ is determined by the density $f(\tau)$ of the distribution of intervals $\tau$ between successive events $[1,2]$,

$$
h(t)=\sum_{n=1}^{\infty} f_{n}(t)
$$

where $f_{1}(t) \equiv f(t)$ and for $n \geq 2$ functions $f_{n}(t)$ are given by the recursive convolution

$$
f_{n}(t)=\int_{0}^{+\infty} f_{n-1}(x) f(t-x) d x .
$$

Qualitatively, functions $f_{n}(t)$ are the distribution densities of so-called $n$-th order intervals between events [2]: denoting as a first-order interval the elapsed time from some given event to the next following event, the second-order interval is defined as the elapsed time between the given event and the second following event, etc. An $n$-th order interval is therefore the sum of $n$ consecutive first-order intervals and is spanned by $(n+1)$ consecutive events.

At asymptotically large time $t \rightarrow+\infty, h(t)$ is saturated [1],

$$
\lim _{t \rightarrow+\infty} h(t)=\rho
$$

Here $\rho$ is the mean rate of events, defined as the inverse mean interval between the successive events,

$$
\rho^{-1}=\int_{0}^{+\infty} \tau f(\tau) d \tau
$$




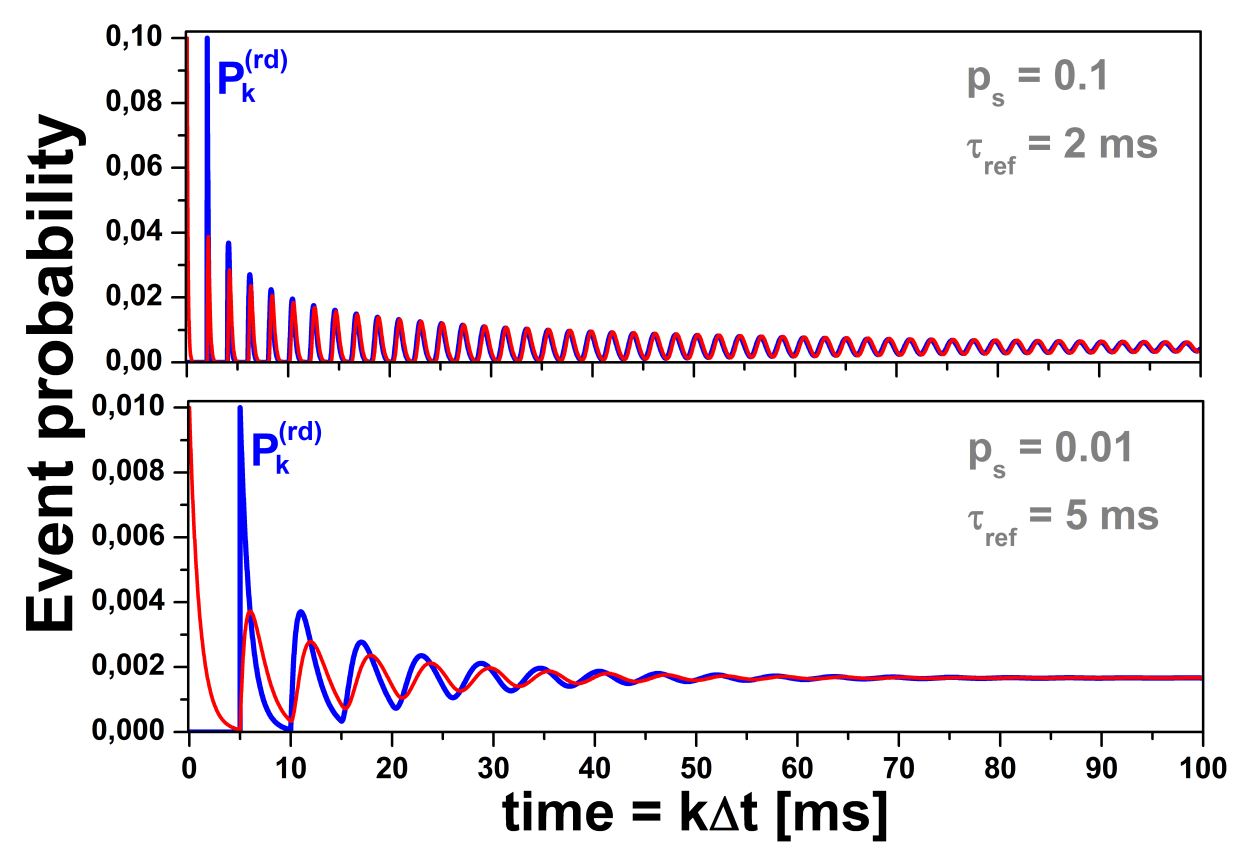

Figure 3: Comparison of the time dependencies for the event probability $P_{k}$ calculated by mutually equivalent formulas (4), (9), (15), (20), (21) in Section 3 (red curves) and the event probability $P_{k}^{(r d)}$ calculated within the renewal theory approach by formula (37) in Section 5 (blue curves). The red and blue curves, accurate to an offset equal to the refractory period, completely coincide. The offset arises due to the different initial condition (exit from the refractory period for the red curves and spike generation for the blue ones) and is left intentionally in order to make the curves distinguishable. Top plot is for $p_{s}=0.1$ and $\tau_{\text {ref }}=2 \mathrm{~ms}$. Bottom plot is for $p_{s}=0.01$ and $\tau_{r e f}=5 \mathrm{~ms}$.

There are many, likely independent, examples of applying the renewal theory results to the case, where the events occurrence is the Poisson process modulated by the constant time of inoperativeness (dead time or, in our notations, absolute refractory period $\tau_{\text {ref }}$ ) or, equivalently, the distribution density of intervals between events has the form of a displaced exponential distribution,

$$
f(t)=\nu_{0} \exp \left(-\nu_{0}\left(t-\tau_{r e f}\right)\right) \theta\left(t-\tau_{r e f}\right)
$$

where $\nu_{0}=p_{s} / \triangle t$ and $\theta(\ldots)$ is the Heaviside step function. In particular, to the best of our knowledge, the first relevant paper dates back to 1947 [5] and has been followed by both in-depth studies [1, 6-9, 12-14] and applied studies for neuroscience [2, 10, 11, 15-20] (see also [21] and [22]). Below, we briefly outline and compare the previous results with our findings.

For $f(t)$ given by (42), using the Laplace transform, one can reduce the formula (39) to the following expression

$$
f_{n}(t)=\nu_{0} \frac{\left(\nu_{0}\left(t-n \tau_{r e f}\right)\right)^{n-1}}{(n-1) !} \exp \left(-\nu_{0}\left(t-n \tau_{r e f}\right)\right) \theta\left(t-n \tau_{r e f}\right)
$$


which is the probability density function for the Erlang/gamma distribution, enabling computation of the renewal density $h(t)$ and the sought-for probability $(37)$. The plot of the function $P_{k}^{(r d)}$ with

$$
h(t)=\sum_{n=1}^{n_{\max }} f_{n}(t)
$$

where $n_{\max }=10^{3}$ and $f_{n}(t)$ is determined by (43), is shown in Fig. 3. The time dependence, accurate to an offset equal to the refractory period, completely coincides with that of $P_{k}$ calculated by mutually equivalent formulas (4), (9), (15), (20), (21) in Section 3. The offset arises due to the different initial condition: an exit from the refractory period at $t=0$ in our model and an event occurrence at $t=0$ in the standard renewal-density approach.

Finally, using (40), (41) and (42), one gets the asymptotic value of $h(t)$,

$$
\lim _{t \rightarrow+\infty} h(t)=\nu_{0} /\left(1+\nu_{0} \tau_{r e f}\right)
$$

which is consistent with formulas (1) and (8) for $\nu$ and $P_{\infty}$, respectively.

\section{Collation with spiking activity data for real neurons}

Though stochastic spiking activity of the neuron serves only as an illustration of the obtained mathematical results, it seems worthwhile to provide the reader with references to experimental data and to discuss the conformity.

Qualitatively, the model studied in the paper can be applied to the case where the neuron is externally stimulated by a relatively strong direct current implemented via a standard patchclamp interface. The spike train variability-inducing noise could be either added to the stimulating current or be naturally ascribed to fluctuations of the neuronal potential due to incoming synaptic signals, imperfect ion channels etc. Alternatively to the stimulation by current, the neuron can have receptive field subject to an intense and nearly constant stimulus (a similar case of the fly photoreceptor is considered in [23], see Figs. 5 and 6 there). Finally, the neuron can be a pacemaker with spontaneous high-frequency tonic spiking activity. For the reference, some examples of relevant experimental data are as follows: Fig. 4 in [24], Fig. 1 in [25], Figs. 1, 3, 4, 5, and 6 in [26], Fig. 3 in [27], Fig. 2B in [28], Figs. $2 \mathrm{H}$ and $8 \mathrm{E}$ in [29], Figs. $4 \mathrm{~A}$ and $4 \mathrm{~B}$ in [30], Fig. 2A in [31], Fig. 4A in [32], and Fig. 4A in [33].

In turn, a quantitative comparison of the obtained results with the experimental findings is hindered by such additional factors as (i) the relative refractory period, which may be time-dependent, and (ii) uncontrollable interaction of the neuron with the surrounding cells [34, 35]. These factors 
often have a dominant influence on the heights, widths and locations of the experimental autocorrelogram peaks, especially if the stimulus magnitude is relatively small. In general, the damped oscillations studied in this paper are well-pronounced only if the absolute refractory period is a dominantly large timescale in the system's dynamics responsible for the event occurrence.

It should also be noted that in most cases of multi-unit systems, such as neuronal networks with neurons as the units, the absolute refractory period is not exactly the same for each unit. For instance, it may be randomly distributed so that each neuron has its own value of the absolute refractory period (e.g., see Fig. 2a in [36]). Alternatively, the absolute refractory period could be updated randomly after each event. Then increasing the variance of the refractory period distribution would apparently blur the damped oscillations. Solving this problem was beyond the scope of the present paper.

\section{Conclusion}

The model considered in this paper is a Bernoulli scheme supplemented by the condition of absolute refractoriness. This formally refers to the renewal theory. However, being quite simple, the model allows obtaining useful results without invoking this formalism. In particular, four equivalent analytical descriptions of the damped oscillations of the event probability have been given: (i) recurrent formula (4) through the difference of two sums, (ii) closed recurrent formula (9), (iii) explicit formula (15) in the form of a polynomial, and (iv) recurrent convolution-type formulas (20), (21). It has also been shown that for the Poisson approximation these results accurately coincide with that of the renewal theory. Finally, using the closed recurrent formula, the relative damping coefficients for the second and third peaks of the event probability have been found in the exact analytical form.

It should be noted that a model analogous to that has been considered in this paper was previously briefly discussed in [37], where a recurrent formula for the event probability, similar to the formula (4), was presented without derivation.

One of the authors (A.V.P.) thanks Evgeny Z. Meilikhov, Laureline Logiaco, and Dylan Festa for stimulating discussions.

[1] D.R. Cox, Renewal Theory (John Wiley \& Sons Inc., New York, 1962).

[2] D.H. Perkel, G.L. Gerstein, G.P. Moore, Neuronal spike trains and stochastic point processes. I. The single spike train, Biophys. J. 7, 391-418 (1967). https://doi .org/10.1016/S0006-3495(67)86596-2 
[3] H.R. Wilson, J.D. Cowan, Excitatory and inhibitory interactions in localized populations of model neurons, Biophys. J. 12, 1-24 (1972). https://doi.org/10.1016/S0006-3495(72)86068-5

[4] O. Kinouchi, M. Copelli, Optimal dynamical range of excitable networks at criticality, Nat. Phys. 2, 348-351 (2006). https://doi.org/10.1038/nphys289

[5] S. Malmquist, A statistical problem connected with the counting of radioactive particles, Ann. Math. Stat. 18, 255-264 (1947). https://doi.org/10.1214/aoms/1177730441

[6] L.M. Ricciardi, F. Esposito, On some distribution functions for non-linear switching elements with finite dead time, Kybernetik 3, 148-152 (1966). https://doi.org/10.1007/BF00288925

[7] J.W. Muller, Dead-time problems, Nucl. Instrum. Methods 112, 47-57 (1973). https : //doi.org/10.1016/0029-554x(73)90773-8

[8] J.W. Muller, Some formulae for a dead-time-distorted poisson process, Nucl. Instrum. Methods 117, 401-404 (1974). https://doi.org/10.1016/0029-554X (74)90283-3

[9] B.I. Cantor, M.C. Teich, Dead-time-corrected photocounting distributions for laser radiation, J. Opt. Soc. Am. 65, 786-791 (1975). https://doi.org/10.1364/JOSA.65.000786

[10] D.H. Johnson, A. Swami, The transmission of signals by auditory-nerve fiber discharge patterns, J. Acoust. Soc. Am. 74, 493-501 (1983). https://doi.org/10.1121/1.389815

[11] D.H. Johnson et al., The application of a point process model to the single unit responses of the cat lateral superior olive to ipsilaterally presented tones, Hearing Res. 21, 135-159 (1986). https : //doi .org/10.1016/0378-5955(86)90035-3

[12] S. Pomme, Time-interval distributions and counting statistics with a non-paralysable spectrometer, Nucl. Instrum. Methods Phys. Res. A 437, 481-489 (1999). https://doi.org/10.1016/S0168-9002(99)00791-3

[13] B. Picinbono, Measurements of second-order properties of point processes, IEEE Trans. Instrum. Meas. 57, 548-555 (2008). https://doi.org/10.1109/TIM.2007.911690

[14] M. Deger et al., Nonequilibrium dynamics of stochastic point processes with refractoriness, Phys. Rev. E 82, 021129 (2010). https://doi.org/10.1103/PhysRevE.82.021129

[15] M. Deger et al., Statistical properties of superimposed stationary spike trains, J. Comput. Neurosci. 32, 443-463 (2012). https://doi.org/10.1007/s10827-011-0362-8

[16] U. Ferrari et al., A simple model for low variability in neural spike trains, Neural Comput. 30, 3009-3036 (2018). https://doi.org/10.1162/neco_a_01125

[17] M.C. Teich, L. Matin, B.I. Cantor, Refractoriness in the maintained discharge of the cat's retinal ganglion cell, J. Opt. Soc. Am. 68, 386-402 (1978). https://doi.org/10.1364/J0SA.68.000386

[18] K. Jones, A. Tubis, E.M. Burns, On the extraction of the signal-excitation function from a non-Poisson cochlear neural spike train, J. Acoust. Soc. Am. 78, 90-94 (1985).

https://doi.org/10.1121/1.392458

[19] W.R. Softky, C. Koch, The highly irregular firing of cortical cells is inconsistent with temporal integration of random EPSPs, J. Neurosci. 13, 334-350 (1993). 
https://doi.org/10.1523/JNEUROSCI.13-01-00334.1993

[20] M.J. Berry, M. Meister, Refractoriness and neural precision, J. Neurosci. 18, 2200-2211 (1998). https://doi.org/10.1523/JNEUROSCI.18-06-02200.1998

[21] D.H. Johnson, Point process models of single-neuron discharges, J. Comput. Neurosci. 3, 275-299 (1996). https://doi.org/10.1007/BF00161089

[22] W. Gerstner, W. Kistler, Spiking Neuron Models: Single Neurons, Populations, Plasticity (Cambridge University Press, 2002). Section 5.2.5. https://doi.org/10.1017/CB09780511815706

[23] Z. Song, M. Juusola, A biomimetic fly photoreceptor model elucidates how stochastic adaptive quantal sampling provides a large dynamic range, J. Physiol. 595, 5439-5456 (2017). https://doi.org/10.1113/JP273614

[24] R.W. Rodieck, Maintained activity of cat retinal ganglion cells, J. Neurophysiol. 30, 1043-1071 (1967). https://doi.org/10.1152/jn.1967.30.5.1043

[25] P.R. Gray, Conditional probability analyses of the spike activity of single neurons, Biophys J. 7, 759-777 (1967). https ://doi.org/10.1016/S0006-3495(67)86621-9

[26] G.P. Moore et al., Statistical signs of synaptic interaction in neurons, Biophys. J. 10, 876-900 (1970). https://doi.org/10.1016/S0006-3495(70)86341-X

[27] J.G. Robson, J.B. Troy, Nature of the maintained discharge of Q, X, and Y retinal ganglion cells of the cat, J. Opt. Soc. Am. A 4, 2301-2307 (1987). https://doi.org/10.1364/JOSAA.4.002301

[28] A. Nini et al., Neurons in the globus pallidus do not show correlated activity in the normal monkey, but phase-locked oscillations appear in the MPTP model of parkinsonism, J. Neurophysiol. 74, 1800-1805 (1995). https://doi.org/10.1152/jn.1995.74.4.1800

[29] H. Kita et al., Role of ionotropic glutamatergic and GABAergic inputs on the firing activity of neurons in the external pallidum in awake monkeys, J. Neurophysiol. 92, 3069-3084 (2004). https://doi.org/10.1152/jn.00346.2004

[30] H. Kita et al., Origins of $\operatorname{GABA}(\mathrm{A})$ and $\operatorname{GABA}(\mathrm{B})$ receptor-mediated responses of globus pallidus induced after stimulation of the putamen in the monkey, J. Neurosci. 26, 6554-6562 (2006). https://doi.org/10.1523/JNEUROSCI.1543-06.2006

[31] J.A. Beatty et al., Complex autonomous firing patterns of striatal low-threshold spike interneurons, J. Neurophysiol. 108, 771-781 (2012). https://doi.org/10.1152/jn.00283.2012

[32] K.-Z. Lee et al., Intraspinal transplantation and modulation of donor neuron electrophysiological activity, Exp. Neurol. 251, 47-57 (2014). https://doi.org/10.1016/j.expneurol.2013.10.016

[33] R. Amit et al., Temporal dynamics of saccades explained by a self-paced process, Sci. Rep. 7, 886 (2017). https://doi.org/10.1038/s41598-017-00881-7

[34] O. Avila-Akerberg, M.J. Chacron, Nonrenewal spike train statistics: causes and functional consequences on neural coding, Exp. Brain Res. 210, 353-371 (2011). https ://doi.org/10.1007/s00221-011-2553-y

[35] M.R. Cohen, A. Kohn, Measuring and interpreting neuronal correlations, Nat. Neurosci. 14, 811-819 
(2011). https://doi.org/10.1038/nn.2842

[36] M. Avissar et al., Refractoriness enhances temporal coding by auditory nerve fibers, J. Neurosci. 33, 7681-7690 (2013). https://doi .org/10.1523/JNEUROSCI . 3405-12. 2013

[37] I. Bar-Gad, Y. Ritov, H. Bergman, The neuronal refractory period causes a short-term peak in the autocorrelation function, J. Neurosci. Methods 104, 155-163 (2001).

https : //doi .org/10.1016/S0165-0270(00)00335-6 